\title{
Alterations in Semen Quality by Antioxidants in Stressed Healthy Individuals
}

\author{
Saurabh Saha ${ }^{1}$, Sonali Saxena ${ }^{2}$, R.K. Srivastava ${ }^{3}$, Chitra Srivastava ${ }^{4}$ \\ ${ }^{1}$ Associate Professor (Physiology), ${ }^{2}$ Associate Professor (Medicine), ${ }^{3}$ Professor (Anatomy), \\ ${ }^{4}$ Associate Professor (Physiology), Department of Physiology, G.S.V.M Medical College, Kanpur
}

\begin{abstract}
The study was conducted to evaluate the role of antioxidants in current therapeutic use on stress induced by exercise on sperm profile. 68 unmarried healthy medical students between 18-24 years of G.S.V.M Medical College who with informed written consentacted as volunteers for study duration of 90 days. Their identities were strictly confidential and were selected randomly. Complete and fresh specimen semen was collected by accepted method of masturbation after abstinence for more than 3 days. Two samples were taken first on commencement and second on conclusion of 3 monthperiod. The subjects have been divided into 4 groups of 17 subjects in a single blinded study. Group I- control and were administered placebo capsules containing arrow root powder. Group II-administered placebo capsules and performed moderate exercise daily for 30 minutes for 3 months on a bicycle ergo meter with persistent increase in heart rate 30 to 50 per minute above their basal heart rate. The subjects carried out exercised for 30 minutes or exercise was stopped due to pain. Group III- carried out the exercise with the same protocol as in group II and were given 1 capsule daily of antioxidant preparation, ECarotin (Franco Indian Pharmaceuticals, India) composition Beta carotene $10 \mathrm{mg}$, vitamin E acetate I.P. $25 \mathrm{mg}$, Vitamin C I.P. $150 \mathrm{mg}$, Selenium dioxide monohydrates U.S.P. $75 \mathrm{mcg}$, Zinc sulphate monohydrate U.S.P. $61.8 \mathrm{mg}$ (equivalent to elemental zinc $22.5 \mathrm{mg}$ ). Group IV - Subjects of this group did not perform any exercise and were administered 1 capsule daily of ECarotin. The following physical features of the ejaculate were recorded Color, Liquefaction Time, $\mathrm{pH}$, Volume Microscopic Examination included Sperm Count, Motility, Sperm morphology while Biochemical estimation done for seminal fluid Fructose. A statistically significant $(\mathrm{p}<.005)$ decrease in sperm countin group II (exercising and taking placebo) and group III (exercising and taking [ECarotin]) but group IV taking only (ECarotin) showed a significant increase in sperm count $(\mathrm{p}<0.05)$. Group's III and IV consuming antioxidant (ECarotin) showed a significant increase in the number of motile sperms $(p<0.05)$ and a significant decrease in the number of non-motile/dead sperms $(\mathrm{p}<0.05)$. Most of the subjects had pus cell in mild to moderate quantity.
\end{abstract}

Keywords: Antioxidants, stress, Sperm count, Sperm Motility, Sperm morphology.

\section{Introduction}

Stress in modern times is a multidimensional omnipresent disease creating issue not addressed by most of us unless disease is established. Infertility cases are increasing in the society resulting in a plethora of

\footnotetext{
Corresponding Author:

Dr. Chitra Srivastava

Associate Professor, Department of Physiology,

G.S.V.M. Medical College, Kanpur

e-mail: drchitrasrivastava@gmail.com
}

anti-fertility treatment options available. The seminal profile has been shown to be influenced by numerous physical as well as psychological factors. One of the important conditions is stress, which has marked influence over quality and quantity of sperm production. However, the role of antioxidants in current therapeutic use has been sparingly explored on semen quality of humans. In the literature, the seminal profile has not been explored in response to exercise producing transient stress. Moreover, the effect of antioxidants has not been explored on stress induced changes in sperm profile. The present study has therefore been aimed to explore the effects of antioxidants on quality and quantity of sperm 
production and also to find out their effects on stress induced changes in sperm profile of human volunteers.

\section{Materials and Method}

Present study has been conducted on 68 unmarried healthy male medical student between 18-24 years. Their identities remain strictly confidential and had been selected randomly for the study. Consent was taken in format as prescribed and ethical clearance was given by ethical committee.

The following anthropometric parameters like height, weight, BMI, pulse rate, blood pressure, and respiratory rate were recorded. Complete and fresh semen specimen was collected from volunteers through accepted method of masturbation with abstinence period of 3 days.

The specimen was obtained in a sterilized, wide month, screw capped containers, to avoid contamination. The time and date of collection was registered on the containers and preserved at standard temperature, physical and microscopic examination on the same day

The following physical features of the ejaculate were recorded Color, Liquefaction Time, $\mathrm{pH}$, Volume Microscopic Examination included Sperm Count, Motility, Sperm morphology while Biochemical analysis of seminal fluid was done for Fructose estimation. The Volunteers have been divided into 4 groups; each group containing 17 subjects in a single blinded study.

Group I - Volunteers in this group served as control and were administered placebo capsules containing arrow root powder for duration of 3 months. Group II - Volunteers in this group performed moderate exercise daily for 30 minutes for 3 months on a bicycle ergometer until they refused to continue exercising, any further on account of fatigue. Heart rate during exercise monitored persistently 30 to 50 per minute above their normal pulse rate. Generally the subjects carried out fast exercise for 7 to 10 minutes slowing down for rest, repetitively until had to stop due to fatigue/pain. Subjects of this group were also given arrow root powder placebo capsules.

Group III - Volunteers in this group carried out the exercise with the same protocol as in group 2 and were given 1 capsule daily of antioxidant preparation, ECarotin (Franco Indian Pharmaceuticals, India) containing Beta carotene $10 \mathrm{mg}$, vitamin E acetate I.P. $25 \mathrm{mg}$, Vitamin C I.P.150 mg, Selenium dioxide monohydrates U.S.P.
$75 \mathrm{mcg}$, Zinc sulphate monohydrate U.S.P. $61.8 \mathrm{mg}$ (equivalent to elemental zinc $22.5 \mathrm{mg}$ ).Group IV Volunteers of this group did not perform any exercise and were administered 1 capsule daily of ECarotin for 3 months.

The average value and their standard deviations of each parameter were calculated. The significance of different between the standard deviations of means in different sets of observations would be assessed at 95\% level of confidence, by applying Student's ' $t$ ' test $(\mathrm{p}<$ 0.05 ) The different groups in any study for 3 months are as follows

\section{The above groups have been compared as follows in the presentwork:}

Group I \& Group IV - This comparison has been carried out to reveal whether ECarotin alone can influence the seminal profile.

Group I \& Group II - This comparison has been necessary to find out if the exercise stress alone has a significance on semen profile.

Group II \& Group III - This comparison has been done to explore whether the intake of ECarotin for 3 months can influence the exercise response (as revealed by comparison of Group I and Group II).

\section{Observations and Results}

The average age of the group is $19.56+0.62$ years. The color of the ejaculate was found to be light cream or yellowish in $10 \%$ of the participating subjects while in $90 \%$ of the subjects it was white or off-white. The odour was typically of fishy type in all the subjects. The viscosity was watery or watery-mucoid in most of the subjects while in some it was found to be thick mucoid.

\section{After 3 months of study:}

Volume: semen in all the four groups showed insignificant change and was within normal range in the initial samples taken and post analysis after 3 months of study. Statistically the 'p' value was insignificant.

pH: The $\mathrm{pH}$ in various groups was in alkaline range and the ' $p$ ' value was insignificant.

Liquefaction Time: The Liquefaction Time in the group I (Control group taking placebo) and group IV (taking antioxidants (ECarotin) only) volunteers showed no significant change in the liquefaction time.But the 
group's doing exercise irrespective of antioxidant (ECarotin) or placebo showed a statistical significant increase in liquefaction Time.

Sperm Count: The sperm count in the group I of control subjects taking placebo showed no significant change, group II (exercising group taking placebo) and group III (exercising group taking antioxidants [ECarotin]) showed a statistically significant decreased $(\mathrm{p}<0.05)$ while group IV taking only antioxidants (ECarotin) showed a significant increase in sperm count $(\mathrm{p}<0.05)$.

Motility: The motility of the sperm was studied after 2 hours of semen liquefaction, which was classified as motile, sluggishly motile and non-motile. The group's III and IV consuming antioxidant (ECarotin) showed a significant increase in the number of motile sperms $(p<0.05)$ and a significant decrease in the number of non-motile/dead sperms $(\mathrm{p}<0.05)$. The group 1 (control students taking placebo) and the group II (exercising students taking placebo) volunteers showed a statistically non-significant change in motility.

Morphology: The morphology of the sperms were categorized into normal and abnormal forms. The abnormal forms seen were round head, pin head, bifurcated head, long tapering heads, bifurcated tail, kinked tail,no tail and lack of acrosomal cap. After 3 months of study there was no significant change in morphology in any group.

Miscellaneous examinations of semen: The pus cells were 3-8 cells per HPF in $98.4 \%$ of the volunteers in the initial sample taken but after 3 months of study $82.6 \%$ of the volunteers showed 3-5 pus cells/HPF. However only 2 volunteers had $20-33$ pus cells/HPF in initial sample which decreased to $8-12$ pus/HPF in the sample taken after 3 months. The semen fructose level was found to be between 263 to $489 \mathrm{mg} \%$, which is within normal range. The clumping of sperms was present in the sample of 8 subjects $(++++)$. Only $18 \%$ of the subjects revealed epithelial cells as 1-2/H.P.F. in initial sample which increased to 3-4/H.P.Fafter 3 months of study. No Trichomonas was seen in any sample.

Some interesting findings were observed during the questionnaire session of the students after 3 months of study.

Vitality (Feeling better): Maximum number of volunteers taking antioxidants reported that they felt better than before and many wanted to continue the preparation being given to them.

Appetite: Maximum number of students taking placebo reported an increase in appetite while most of the students taking antioxidants reported no change in appetite behavior.

Memory: Some of the students taking antioxidants reported a better retention power.

Sleep: A large number of students taking placebo gave history of increased desire to sleep while most of the group of students taking antioxidants reported no alteration in sleep pattern.

Desire for sex/Libido: There was a significant increase in the number of volunteers who gave history of increased desire for sex/increased libido who were taking antioxidants. Less so in the exercising group but more in the group taking antioxidants only.

\section{Discussion}

In the present study, we found that the volume of the ejaculate in most volunteers averaging between $2.3 \mathrm{ml}-$ $3.0 \mathrm{ml}$, which is in accordance with the reported values of Falk and Kaufman ${ }^{[1]}$ (1950), Bhushan et al (1978)[2] range of volume was ranging from 1.0-7.2 ml.

The $\mathrm{pH}$ of seminal fluid was found to be alkaline (8.4-8.6) in nature which is similar to findings of Bhushan et al (1978) ${ }^{[2]}$ in Indian subjects.

The average Liquefaction time in our study was found at an average to be between 14.4-18.7 minutes, which corroborates with the findings of Lunenfeld and Glezerman (1981) ${ }^{[3]}$.

The sperm counts, in our study, in the exercising individuals were noted to be decreased. These changes may be attributed to the negative effects of stress produced due to exercise because of the generation of reactive oxygen species. Our findings also corroborates with publication of JAMA (1988) ${ }^{[4]}$ in which Vietnam Veterans had decreased sperm count and also decreased number of morphologically normal sperms due to war-psychology. Fenster L et al (1997) ${ }^{[5]}$ and Clarke RN et al (1999) ${ }^{[6]}$, both of the studies showed an inverse relationship between semen quality and the stress produced due to emotionally charged situations. Comhaire FH et al (2000) ${ }^{[7]}$ who observed that vitamin 
A and vitamin E supplementation decreases the reactive oxygen species in oligozoospermic men. We have found a decrease in sperm count in the exercising individuals while an increase in sperm count in the individuals taking antioxidants. This finding is supported by observations made by Clarkson PM et al $(2000)^{[8]}$ who observed that exercise increases reactive oxygen species but trained athletes receiving antioxidant supplements showed evidence of decreased oxidative stress.

The motility of the sperms in our study was found to be significantly increased while the number of nonmotile sperms decreased significantly after taking antioxidants. Our findings of increase in motility is supported by reports of Omu AE et al (1998) ${ }^{[9]}$ in which they suggested that Zinc increases the motility and the sperm count in men with asthenozoospermia while Scott R et al (1998) ${ }^{[10]}$ who emphasized that Selenium increases motility in sub-fertile men.

In our study, we found that the semen contained a higher percentage of normal and a lower percentage of morphologically abnormal sperms and there was no significant difference between the initial and the final samples. This is supported by the study of Bartoov B et al $(1981,1982)^{[11]}$ in which observed that the morphologically normal sperms have a frequency of $58 \%$ or more as found in our study. The semen fructose levels in our study were found to be within normal range (243-489 $\mathrm{mg} \%$ as recommended by Eliasson and Treichl (1971) ${ }^{[12]}$ who reported semen fructose levels to be normal within $150-600 \mathrm{mg} \%$ range. The pus cells in the semen sample of north Indian males are indicative of sub-clinical infection for which they do not take any treatment.

\section{Conclusion}

The volume of the ejaculate in the volunteers showed no significant change in the initial sample or after 3 months of study in all the groups.

The $\mathrm{pH}$ of the ejaculate in the volunteers showed no significant change in both the samples and in all the groups suggesting that antioxidants have no effect of on $\mathrm{pH}$.

The Liquefaction time of semen in the volunteers who were exercising showed a significant increase in it thus demonstrating that exercise has induced a negative effect on liquefaction time. The volunteers who were taking only antioxidants showed no change in liquefaction time.

Antioxidants may increase sperm count but the count of morphologically normal and duration to stay motile increases significantly.

The exercising volunteers who were taking placebo (group II) showed a significant decrease in sperm count due to oxidative stress induced by exercise. The same observation was made in the exercising volunteers taking antioxidants (group III) but the decrease in sperm count was comparatively lesser than group II of volunteers who were doing exercise and taking placebo. So, antioxidants negate the effect of exercise induced stress.

Maximum percentage of North Indian males were having normal sperm count. Exercise induced stress can lead to the normal sperm count of the individuals at the lower normal range (20-36 million/ml) to an oligospermic state. So, exercise led to a significant decrease in sperm count.

The motility of sperms increased significantly after taking antioxidants while the number of non-motile sperms decreased significantly after taking antioxidants.

Maximum number of North Indian males showed pus cells in the semen sample indicative of sub-clinical infection.

The semen fructose level in North Indian subjects was found to be within normal range.

Desire for sex/libido, feeling better and the memory was reportedly better in the volunteers taking antioxidants.

\section{Conflict of Interest: Nil}

\section{Source of Funding: Self}

Ethical Clearance: Study was approved by Ethical Committee.

\section{References}

1. Falk HCand Kaufman SA. What constitutes normal semen. Fertil. Steril. 1950; 1:489.

2. Bhushan S, Pandey RC, Singh SP. Some observations on human semen analysis. Ind. J. Physiol Pharmacol. 1978; 22:393-396.

3. Lunenfeld B, and Glezerman $M$. Diagnose und therapie manulicher fertilitats storungen. Grosse.1981; Pg 23. 
4. JAMA. Health status of Vietnam veterans. 1988; 259:2708-2714.

5. Fenster L, Katz DF. Effects of psychological stress on human semen quality. J. Androl.1997; 18:194202.

6. Clarke RN, Klock SC, Geoghegan A, Travassos DE. Relationship between psychological stress and semen quality among in-vitro fertilization patients. Hum. Reprod. 1999; 14:753-758.

7. Comhaire FH, Christophe AB, Zalata AA, DhoogeWS, MahmoudAM. The effects of combined conventional treatment, oral antioxidants and essentialfatty acids on sperm biology in subfertile men. Leukot. 2000; 63:159-165.

8. Clarkson PM, Thompson HS. Antioxidants: what role do they play in physical activity and health? Am. J. Clin. Nutr. 2000; 72(2 Suppl):637-646.

9. Omu A, Dashti H and Al-Othman S. Treatment of asthenozoospermia with zincsulphate,androlog ical,immunological and obstetric outcome. Eur. J. Obstet. Gynecol. Reprod. Biol. 1998; 79:179-184.

10. Scott R, MacPherson A, Yates RW, Hussain B, and Dixon J. The effect of oral selenium supplementation on human sperm motility. British. J. Urol. 1998; 82:76-80.O

11. Bartoov B, Eltes F, and PanskyM. Estimating fertilization potential via semen analysis data. Hum. Reprod. 1993; Aug: 65-70.

12. Eliasson R. and TreichlL. Supravital staining of human spermatozoa. Fertil. Steril. 1971; 22:134. 